\title{
Capturing complexity: Field-testing the use of 'structure from motion' derived virtual models to replicate standard measures of reef physical structure
}

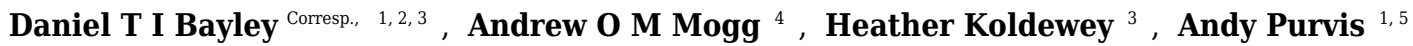 \\ 1 Department of Life Sciences, Natural History Museum of London, London, United Kingdom \\ 2 Centre for Biodiversity and Environment Research, University College London, University of London, London, United Kingdom \\ 3 Conservation Programmes, Zoological society of London, London, United Kingdom \\ 4 Tritonia Scientific, Oban, United Kingdom \\ 5 Department of Life Sciences, Imperial College London, London, United Kingdom \\ Corresponding Author: Daniel T I Bayley \\ Email address: daniel.bayley.14@ucl.ac.uk
}

Reef structural complexity provides important refuge habitat for a range of marine organisms, and is a useful indicator of the health and resilience of reefs as a whole. Marine scientists have recently begun to use 'Structure from Motion' (SfM) photogrammetry in order to accurately and repeatably capture the 3D structure of physical objects underwater, including reefs. There has however been limited research on the comparability of this new method with existing analogue methods already used widely for measuring and monitoring 3D structure, such as 'tape and chain rugosity index' and graded visual assessments. Our findings show that analogue and SfM Rugosity Index can be reliably converted over a standard 10-metre reef section (SFM Rugosity Index $=1.348 \mathrm{x}$ chain Rugosity Index - 0.359, $r^{2}=0.82$; and Chain Rugosity Index $=0.606 \times$ SfM Rugosity Index +0.465$)$ for Rugosity Index values up to 2.0 , however SfM Rugosity Index values above this number become increasingly divergent from traditional tape and chain measurements. Additionally we found SfM Rugosity Index correlates well with visual assessment grades of coral reef over a $10 \times 10$ metre area (SfM Rugosity Index $=0.1461 \times$ visual grade $\left.+1.117 ; r^{2}=0.83\right)$. The SfM method is shown to be affordable and nondestructive whilst also allowing the data collected to be archival, less biased by the observer, and broader in its scope of applications than standard methods. This work allows researchers to easily transition from analogue to digital structural assessment techniques, facilitating continued long-term monitoring, whilst also improving the quality and additional research value of the data collected. 


\section{Capturing complexity: Field-testing the use of 'structure}

\section{2 from motion' derived virtual models to replicate standard}

\section{3 measures of reef physical structure}

4 Authors: Daniel T.I. Bayley ${ }^{1,2,3}$, Andrew O.M. Mogg ${ }^{4}$ Heather Koldewey $^{3}$, Andy Purvis ${ }^{1,5}$

5 Affiliations:

6 1. Department of Life Sciences, Natural History Museum of London, Cromwell Rd, South

$7 \quad$ Kensington, London SW7 5BD, U.K.

8 2. Centre for Biodiversity and Environment Research, University College London, Gower

9 Street, London WC1E 6BT, U.K.

10 3. Zoological Society of London, Regent's Park, London, England NW1 4RY, U.K.

11 4. Tritonia Scientific, Dunstaffnage Marine Laboratories, Oban PA37 1QA, U.K.

12 5. Department of Life Sciences, Imperial College London, Silwood Park campus, Ascot SL5 7PU, U.K.

Correspondence e-mail: Daniel.Bayley.14@ucl.ac.uk

\section{Abstract}

17 Reef structural complexity provides important refuge habitat for a range of marine organisms,

18 and is a useful indicator of the health and resilience of reefs as a whole. Marine scientists have

19 recently begun to use 'Structure from Motion' (SfM) photogrammetry in order to accurately and 
20 repeatably capture the 3D structure of physical objects underwater, including reefs. There has

21 however been limited research on the comparability of this new method with existing analogue

22 methods already used widely for measuring and monitoring 3D structure, such as 'tape and chain

23 rugosity index' and graded visual assessments. Our findings show that analogue and SfM

24 Rugosity Index can be reliably converted over a standard 10-metre reef section (SfM Rugosity

25 Index $=1.348 x$ chain Rugosity Index $-0.359, r^{2}=0.82$; and Chain Rugosity Index $=0.606 x$

26 SfM Rugosity Index +0.465 ) for Rugosity Index values up to 2.0, however SfM Rugosity Index

27 values above this number become increasingly divergent from traditional tape and chain

28 measurements. Additionally we found SfM Rugosity Index correlates well with visual

29 assessment grades of coral reef over a $10 \times 10$ metre area (SfM Rugosity Index $=0.1461 \times$ visual

30 grade $\left.+1.117 ; \mathrm{r}^{2}=0.83\right)$. The $\mathrm{SfM}$ method is shown to be affordable and non-destructive whilst

31 also allowing the data collected to be archival, less biased by the observer, and broader in its

32 scope of applications than standard methods. This work allows researchers to easily transition

33 from analogue to digital structural assessment techniques, facilitating continued long-term

34 monitoring, whilst also improving the quality and additional research value of the data collected.

36 Introduction

37 The physical structure of coral reef habitats is a strong determinant of the abundance and

38 diversity of many reef-associated organisms (Graham \& Nash 2013; Darling et al. 2017).

39 Morphologically complex coral structures also indicate a reef's current health and its likelihood

40 of rebounding from disturbance events such as heat-induced bleaching (Alvarez-Filip et al. 2009;

41 Graham et al. 2015). Changes to reef structure can be ecologically relevant at a range of scales 
42 according to the reef-associated organism's body size; therefore, even centimetre-level changes

43 in habitat can be important to reef community structure on a local scale (Nash et al. 2013).

44 Despite the importance of reef structural complexity and its incorporation into many standard 45 reef-monitoring protocols (Bayley \& Mogg 2019), quantification and monitoring of structural 46 changes through time has remained relatively simplistic. Typical monitoring budgets tend to be 47 restrictive, so coarse visual or analogue methods that combine practicability with low cost - such as ‘tape-and-chain rugosity’ (Risk 1972), broad qualitative visual estimation (Wilson, Graham \& 49 Polunin 2007) or depth measures (Dustan, Doherty \& Pardede 2013) - are most commonly used. Structural assessments conducted using such methods tend, however, to be limited in scale due to SCUBA time restraints as they rely on researchers being in the water and are time-consuming to complete (Knudby \& LeDrew 2007; Harborne, Mumby \& Ferrari 2012).

Standard analogue techniques have proven useful for broadly describing reef structure for ecological analysis (Alvarez-Filip et al. 2009; Graham \& Nash 2013; Graham et al. 2015), but are criticised for being highly variable in their results due to recorder bias (Wilson, Graham \& Polunin 2007), and for often giving high variability from even small changes in measure placement. Furthermore, standard topographic measures such as 'rugosity' usually produce only one unbounded linear metric at a single coarse (centimetre) resolution, limiting the usefulness of such measures for describing complex differences in the physical form of individual underwater

60 structures or reefscapes. The use of just one simplistic and poorly-repeatable metric is likely

61 therefore to also limit our ability to relate reef structure to reef fish population sizes and

62 community structure (Knudby \& LeDrew 2007; Nash et al. 2013; Young et al. 2017).

63 Recent advances in technology and computing power are providing new, data-dense and 64 quantitative virtual techniques to measure the 3D structure of objects underwater. The use of 
65 LiDAR, Sonar, and satellite-based technologies to assess benthic structure and bathymetry is 66 now commonplace (Brown et al. 2011), and has revolutionised the measurement of benthic

67 topography, revealing new patterns and interactions in spatial ecology (Brock \& Purkis 2009;

68 Costa, Battista \& Pittman 2009; Purkis 2018). However, such methods are extremely costly to

69 deploy, require specialist training to operate and are restricted by water depth. Furthermore they

70 can only detect features greater than roughly one metre in size (Kenny et al. 2003; Costa,

71 Battista \& Pittman 2009).

72 Land-based methodologies for accurate, cost-effective 3D measurement have been advancing 73 rapidly and are now being adapted for use in aquatic environments. One such approach that has

74 been gaining popularity is 'Structure From Motion' photogrammetry (SfM) (Westoby et al.

75 2012), which creates scaled 3D digitally-derived virtual surface model renderings of objects in

76 fine detail from multiple overlapping photographic or video images and reference markers. The

77 imagery needed for such models can be collected using a single standard underwater camera,

78 with no need for an expensive rig. Once the virtual reef surface has been created and calibrated

79 using open-source or specialist software, detailed morphometric surface analyses can be

80 undertaken on the object of interest.

81 Several studies have recently detailed the application of the SfM technique to underwater marine

82 environments, showing the technique to be useful for quantifying structure across scales from

83 colony to reef-scape (Leon et al. 2015; Burns et al. 2015a; Ferrari et al. 2016b; Teague \& Scott

84 2017; Young et al. 2017). SfM has also been shown to be fast, accurate and repeatable (Lavy et

85 al. 2015; Burns et al. 2015a; Figueira et al. 2015; Storlazzi et al. 2016; Bryson et al. 2017).

86 However, there has only been limited research into whether this new method of assessment can

87 provide measurements that are directly transferable from current standard monitoring protocols 
88 (Ferrari et al. 2016b), meaning it is not yet clear whether ongoing surveys can transition to this

89 new technique without risking the loss of comparability with older survey data.

90 Here, we empirically compare standard structural survey methods for coral reefs with SfM and

91 test whether the resulting data can be inter-calibrated. We compare the SfM technique to the two

92 most widely used standard methods of structure assessment: 'tape and chain rugosity' and

93 graded visual assessment. We use a much larger set of validation transects than previous

94 comparisons (Ferrari et al. 2016b; Young et al. 2017), and apply them over a range of coral reef

95 habitats, at the most widely applied scale of 10 metres. We also discuss SFM's additional

96 possible outputs and its costs and benefits. We argue that SfM increases versatility, repeatability,

97 and archival value of reef surveys.

\section{Materials and Methods}

99

100

101

102

103 (Figure 1).

104 Reef structure was recorded within varying visual grades of habitat complexity over a square 10

$105 \times 10 \mathrm{~m}\left(100 \mathrm{~m}^{2}\right)$ reef area following the method described by Polunin \& Roberts (1993), with 106 grades ranging from 0 to 4 , corresponding to: $0=$ no vertical relief; $1=$ low and sparse relief; $2=$

107 low but widespread relief; 3 = moderately complex; 4 = very complex with numerous caves and

108 fissures. For each survey area the dominant broad substrate type (i.e. $>40 \%$ cover of sand / 
109 rubble / hard coral growth form / soft coral / algae) was also recorded visually (English et al.

110 1997).

111 Inside each $100 \mathrm{~m}^{2}$ area, Rugosity Index (RI) was calculated for $8-10$ evenly-spaced parallel

112 transects using the widely-used 'tape and chain' method (English et al. 1997). A 10-metre brass

113 chain with 1-cm links was moulded to the hard substratum in order to measure the topographic

114 (surface) distance. The direct horizontal distance between the start and end of the chain was then

115 recorded, giving linear distance (Figure 2). Rugosity Index (RI) is then calculated as Surface

116 distance $(S D) /$ linear distance $(L D)$

117 After each chain was laid, a weighted reflective marker of known dimensions (147 x $50 \mathrm{~mm})$

118 was placed at the start and end of the chain and left in place while the chains were removed. This

119 process resulted in a set of 20 fixed start and end markers in each quadrat, indicating LD for each

120 transect (Figure 2).

121 Following the in situ RI measurement, each whole quadrat was imaged across its full area in a

122 lawnmower pattern from a distance of $\sim 2$ metres above the substrate (dependent on visibility),

123 following Burns et al. (2015), to collect multiple overlapping images of the reef, using a Nikon

124 D750 DSLR camera with a wide-angle fixed $20 \mathrm{~mm}$ Nikor lens and dome port. The same camera

125 was used for all imaging, to capture high detail (6016 x 4016 px) images, and to prevent any

126 potential variation between resulting models from differing camera or lens types (Lavy et al.

127 2015). The footprint of a typical photo in this study was approximately $2.5 \mathrm{~m} \times 1.75 \mathrm{~m}$, (though

128 this area can increase and decrease as the camera orientation shifts). Linear overlap of each

129 photograph's footprint varied in line with substrate complexity, but ranged from $75 \%$ to $95 \%$

130 across this study. Sidelap (lateral overlap) ranged from $75 \%$, up to $90 \%$. Overlaps were 
131 calculated post-hoc by placing markers in the middle of images and measuring the linear and

132 lateral distances between image sets.

133 Once images were collected, a digital surface model (DSM) of the area photographed was

134 created using Agisoft 'Photoscan' Professional (now Agisoft 'Metashape') software (Agisoft

135 LLC 2017). Each $100 \mathrm{~m}^{2}$ model was based on the alignment of approximately $600-1000$

136 overlapping digital photographs, dependent upon light levels and structure of the benthic

137 topography (alignment settings = high accuracy, generic pre-selection of images, 40,000 key-

138 point limit, 10,000 tie-point limit). This process typically gave a dense XYZ point-cloud of

139 around 4,000 matched points per $\mathrm{m}^{2}$. The dense point-cloud was then converted into a Delaunay

140 Triangulated Irregular Network (TIN) wireframe mesh, with medium quality reconstruction,

141 aggressive depth filtering and standard interpolation settings applied. Finally, using tiled imagery

142 overlaid onto the filled reef mesh, ten of the in-situ reflective markers' lengths were used to

143 calibrate each model to these known distances $(147 \mathrm{~mm})$ in xyz space, giving overall averaged

144 scaling accuracies of $<5 \mathrm{~mm}$. Approximate depth ( $\mathrm{Z}$ dimension) of the first marker in each

145 quadrat was measured using a Suunto Gekko dive computer, and the models were orientated to a

146 level XY plane using an in-situ spirit-level.

147 The xyz pointclouds of each rendered reefscape were exported from Photoscan and analysed for

148 RI metrics within Gwyddion software (Nečas \& Klapetek 2012), using the surface roughness

149 analysis tool, following rasterization (at $1 \mathrm{~cm}$ pixel resolution, with averaged linear point

150 interpolation, plus mirrored exterior), and surface distance measurement with zero cut-off

151 (Figure 3). Surface point values were averaged across 10 pixels to account for any fine-scale

152 deviation of the in situ chain from the perfectly straight virtual transect line used in analysis. 


\section{Data Analysis}

154 Linear regression was used to relate the matched linear distances and RI values $(n=58)$ from in

155 situ and virtual transects. If both methods give very similar RI estimates, the regression between

156 them is expected to have an intercept of 0 and a slope of 1; Student's t-tests were used to assess

157 whether the data fitted this expectation. We also used linear regression to relate the in situ and

158 virtual measures of RI to visual reef estimation techniques (Polunin \& Roberts 1993) on a five

159 point scale $(0-4)$. Analysis of covariance was used to test whether the relationships between

160 virtual and in situ RI varied among dominant substrate types. We explored log-transformation of

161 RI estimates because, by inspection, variances were higher at higher values of RI; but

162 transformation did not fully remedy this and made very little difference to goodness-of-fit, so we

163 report the results from the untransformed data. All analyses were carried out using the $\operatorname{lm}$

164 function in R, version 3.4.1 (R Core Team 2016).

\section{Results}

\section{Tape \& Chain method}

167 In situ (chain-measured) and virtual (SfM-derived) values of linear distance were practically

168 identical $\left(\mathrm{R}^{2}=0.9994\right.$, mean difference $=0.002, \mathrm{SD}=0.02 \mathrm{~m}$; all mean differences are reported

169 as virtual - in situ measurement), as expected. Comparison of surface distance between the same

170 points for the in situ chain-measured distance of 10 metres and the virtually measured distance

171 gave good agreement within simple substrates i.e. rubble fields (Mean difference $=0.01, \mathrm{SD}=$

$1720.29 \mathrm{~m})$, foliose dominated reefs $($ Mean difference $=0.17, \mathrm{SD}=0.44 \mathrm{~m})$, and mixed table /

173 massive corals on sand (Mean difference $=0.12, \mathrm{SD}=0.33 \mathrm{~m}$ ). There was, however, less

174 agreement between the methods within more complex habitats, with higher variance and with

175 virtual distances tending to be larger than in situ measurements (Figure 4) i.e. dense branching 
176 Acropora thickets (Mean difference $=1.41, \mathrm{SD}=0.74 \mathrm{~m}$ ), and mixed corymbose and massive

177 growth form communities (Mean difference $=1.39, \mathrm{SD}=0.59 \mathrm{~m}$ ).

178 Virtual RI was well-predicted by in situ SfM RI in the regression $\left(\mathrm{R}^{2}=0.82\right.$; Figure 5).

179 However, the intercept differed significantly from zero (estimate $=-0.359, \mathrm{t}=-3.095,56$ d.f., $\mathrm{p}=$

1800.003 ) and the slope differed significantly from 1 (estimate $=1.348, \mathrm{t}=15.78,56 \mathrm{~d} . \mathrm{f} ., \mathrm{p}<$

181 0.001), because values for virtual RI gradually become greater for every corresponding value of

182 in-situ RI as surfaces become more complex. The regression equations for converting between in

183 situ and virtual estimates of RI are:

Inclusion of dominant substrate type as a factor increased the predictive ability of the regression

$187\left(\right.$ ANCOVA; $\left.F_{6,51}=104.6, p<0.001, R^{2}=0.92\right)$, demonstrating that the level of substrate

188 complexity affects the accuracy of the RI comparisons.

\section{Visual estimation method}

190 In situ and SfM-based methods both fit broadly to the visual assessment method proposed by

191 Polunin \& Roberts (1993) (Figure 6). However, the fit was markedly better for the SfM virtual

192 method $\left(\mathrm{R}^{2}=83.1 \%, \mathrm{~F}_{1,56}=274.7, \mathrm{p}<0.0001\right)$ than for the in-situ tape and chain method $\left(\mathrm{R}^{2}=\right.$

$\left.19366.3 \%, \mathrm{~F}_{1,56}=110.4, \mathrm{p}<0.0001\right)$. The regression equations for converting between virtual or in-

194 situ estimates of RI and visual estimates are: 


\section{Discussion}

\section{Rugosity Index assessment}

199 Surface distances, and hence estimates of Rugosity Index (RI), produced by Structure from

200 Motion photogrammetry (SfM) over coral reefs correlated strongly with those from in situ tape

201 and chain measurement, but SfM values were larger and more variable than tape and chain

202 estimates in more structurally complex reefscapes. There are three likely reasons for the disparity

203 in estimates of surface distance (and therefore RI). Firstly, the models created through SfM

204 become increasingly likely to produce areas of misaligned points as the complexity of the surface

205 of interest increases, even with aggressive filtering of anomalous points from the dense point-

206 cloud. These misaligned points lead to the need for greater post-processing cleaning for highly

207 complex objects, or an expectation of some limited complexity overestimation.

208 The second reason for increased complexity values from the SfM derived method is because soft

209 organisms, such as soft corals, extended polyp tentacles of hard coral, algae, worm feeding

210 appendages and crinoids, all have structure which would not be recorded by the tape and chain

211 method (Ferrari 2017), but they will be recorded by SfM as long as these organisms are

212 stationary during imaging. Relatively still water conditions are therefore needed for accurate

213 assessment of soft structures such as these.

214 Finally, it is likely that the virtual surface created through SfM more accurately reflects the real

215 structural complexity than in situ methods because an in-situ chain will typically fall off of finely

216 branching structures and tend towards the lowest stable points of the reef through gravity. This

217 makes measurement of slender, overhanging or highly complex objects difficult and likely to be

218 under-estimated in terms of their rugosity, even if additional time is spent moulding the chain to 
219 complex / overhanging surfaces. The SfM technique shares this limitation of typically not being

220 able to fully capture overhanging surfaces, however incorporating an increased numbers of

221 oblique angle or upward facing photos where possible (with appropriate lighting) will minimize

222 this limitation and can allow vertical structures to fully overhanging cave systems to be imaged

223 using SfM (Hernández et al. 2016; Robert et al. 2017; De Waele et al. 2018).

224 One shared limitation between the tape and chain and SfM methods for measurement of RI is an

225 inability to fully assess structures such as densely branching growth forms and tightly packed

226 overhanging structures such as terraced table corals. While SfM can capture more of this

227 structure through oblique angled photography and good lighting, it is still limited by what can be

228 seen, so obscured areas within a coral matrix for instance will still not be quantified (Lavy et al.

229 2015). Likewise, good underwater visual conditions are essential for adequate model creation as

230 turbid, low light environments will produce lower quality outputs (Bryson et al. 2017).

\section{Visual complexity assessment}

232 In a similar fashion to the comparison with tape and chain rugosity, SfM Rugosity Index

233 correlated very well to visual estimates of structural complexity over coral reefs, explaining

$23483.1 \%$ of the total regression variance. The SfM method furthermore improves our ability to

235 compare visual complexity to Rugosity Index values, with the visual estimates of structural

236 complexity explaining $23 \%$ more of the variance from correlation to virtual (SfM) RI than from

237 correlation to the in situ (tape and chain) RI. This indicates that SfM better matches how our eye

238 naturally assesses complexity of structures. The reduced coefficient of determination using the

239 chain method was primarily driven by the lack of differentiation in rugosity indices within higher

240 complexity sites ( $2-4$ on the visual scale) and the wider variance of RI values at each grade.

241 While no other studies that we know of directly compare SfM Rugosity Index to visual 
242 assessments of structure, our results do match well with the study by Wilson, Graham \& Polunin

243 (2007), which found visual estimates of reef topography were significantly correlated with tape

244 and chain estimated rugosity.

245 Our analysis furthermore illustrates the pitfalls of taking single measures of RI, as relatively high

246 variance of RI values are observed at each visual grade for both the in situ and virtual techniques.

247 This shows the need for multiple replicates of linear RI to be taken at each site, whichever

248 method is applied, in order to give a reliable approximation of overall structure.

\section{Costs, benefits \& limitations}

250 The concept of 'photogrammetry' has been around as long as photography, but it is only within

251 roughly the last five years that we have reached a point where advances in both digital camera

252 technology and digital processing power have made the SfM technique economically viable and

253 time-effective. The development of fast digital photogrammetry algorithms and the now

254 widespread use of low-cost underwater action cameras such as GoPro ${ }^{\mathrm{TM}}$ opens up a plethora of

255 opportunities in the marine world for recreating virtual benthic formations such as reefs, and

256 enables us to analyse them in an efficient, objective and cost-effective way. These measures

257 allow us to move from the simplistic chain or visual methods to objective and quantitative

258 morphometric analyses of volume, surfaces, traits, and spatial distribution of individual colonies,

259 which can be analysed at the scale of interest to the study. Work has indeed already begun to

260 scale-up this method and integrate the use of this technology with drones to allow much greater

261 area coverage with reduced risks and costs (Chirayath \& Earle 2016), which is likely to

262 revolutionise shallow reef monitoring work.

263 Table 1 details the costs and benefits of each method discussed in this work, in terms of field and

264 processing time, scope of analyses possible, and reproducibility of the analyses. The 
265 comparisons are not comprehensive but give a broad view of the most widely used methods

266 contrasted against Structure from Motion using single or cluster-based processing. In this sense,

267 a 'cluster' is a network of two or more computers acting as linked servers (nodes), essentially

268 allowing greater processing power by combining the resources of each linked computer.

269 Whilst the time costs of initial data collection are comparable for each methodology, the initial

270 processing time of SfM is the primary limiting factor currently, and is explicitly dependent upon

271 the computer processing power available to the researcher. As processing time is reduced,

272 through cluster-based processing, (or through likely future accelerated processing speeds as

273 computing power advances), the range of added benefits from the SfM method become apparent.

274 Agisoft Photoscan is the software currently most commonly used for this type of 3D model

275 generation of reefs, due to its easy interface, range of capabilities, relative low cost, and facility

276 to integrate with workstreams such as Python. However, there are a range of other brands

277 available such as Pix4D, Bundler and Autodesk, with differing specialities but broadly similar

278 capabilities. Post processing measurements can also be completed with a number of different

279 software packages, dependent on the specified objective. Some frequently used packages are

280 ArcGIS '3D analyst', Rhino, and the open-sourced Meshlab and R. We chose to use Gwyddion

281 due to its versatility of functions, easy interface and because it is also open-source with good

282 documentation. While Gwyddion was originally designed for electron microscope surface

283 metrology, the techniques needed to analyse a reef surface are nevertheless the same.

284 There are of course still limitations to the SfM technique within a marine setting. For effective

285 models to be created, the images collected need to be clear and sharp, and this can be a

286 challenge in low-light / turbid / high energy environments. Steady camera work with adequate

287 water clarity and good lighting is therefore essential. One of the benefits of SfM however is its 
288 ability to generate image mosaics far larger than those captured by an individual image. This 289 means that, whilst it is possible to model objects of great size, it is also possible to model objects 290 in very poor visibility, given sufficient image overlap. The relationship between the distance of

291 the camera from the object and the resulting photographic footprint is a linear one, meaning that

292 the footprint will be twice the size at twice the distance. In turn, this means that a survey

293 conducted in poor conditions (e.g. $0.5 \mathrm{~m}$ effective visibility) will require 4 times the number of

294 images to cover just the same photographic width as a single image from a survey conducted at 2

$295 \mathrm{~m}$ effective visibility. This resulting increase in photographic number has a knock-on effect on

296 the computing power and resources need to model a single area, possibly rendering such an

297 effort impractical in poor conditions with current general/mid-level computing systems.

298 Conversely in good visibility conditions, the photographer must make a decision between

299 coverage and detail, as image resolution will be lost the further the camera moves from the

300 substrate (Hitchin, Turner \& Verling 2015).

301 Linked with the issue of adequate visibility is the need to bear in mind obscurement of objects,

302 particularly in dense coral thickets, or from overlapping table growth forms (Goatley \&

303 Bellwood 2011; Figueira et al. 2015). We were able to minimise the error associated with such

304 habitats in this study by increasing the number of images taken in more complex habitats, by

305 incorporating oblique angle photos (whilst ensuring to minimize large blue-water sections in the

306 images), and by maintaining good lighting (Hitchin, Turner \& Verling 2015; Burns et al. 2015b;

307 Pizarro et al. 2017). Despite these considerations, some sections of reef, such as areas of dense

308 coral matrix and highly branching variable height sections where oblique images are restricted,

309 will always have some level of obscurement that will introduce gaps to the model. While these

310 gaps are interpolated in the model building process, they will be of course only be statistical 
311 estimations and this uncertainty must be considered and minimised whenever using this

312 technique.

313 Following on from these stated limitations, this study investigates tropical coral reef habitats

314 only, and not rocky or temperate biogenic reefs. While it is unlikely that any significant

315 differences in the conversion between in situ and virtual measures of structure will occur in these

316 differing habitats given the technique's accuracy (Figueira et al. 2015; Bryson et al. 2017; Raoult

317 et al. 2017), this has not been explicitly tested here. We would therefore recommend future

318 research across these different habitats and conditions, including further investigations into the

319 effects of scale (i.e. colony to reefscape), and fractal dimension / resolution of measurement.

320 Despite these current limitations, our study demonstrates that the SfM technique can be used to

321 easily transition from analogue to digital structural assessment, allowing continued long-term

322 coral reef structure monitoring. Furthermore, the increased range of analyses available from the

323 creation of virtual reefscapes is likely to lead to a clearer understanding of the ecological

324 processes related to reef physical structure. 


\section{Conclusions}

326 The SfM technique shows great promise for future survey efforts due to its ease of use across

327 multiple depths, scales, and reef types as well as its non-destructive nature. The outputs from a

328 single survey can be used in a number of different analyses, for instance virtual transects can

329 easily be applied to currently laborious field methodologies such as estimation of carbonate

330 budgets (Perry et al. 2012), increasing the speed, scale and accuracy of assessments.

331 Additionally, the ability to measure not just hard corals but other often neglected aspects of the

332 reef which provide important structure, such as soft corals or macro-algae, may help give insight

333 into associated community dynamics.

334 Perhaps most importantly however, the SfM method is quantitative, is less biased by the

335 recorder, and is replicable. The technique therefore allows detailed spatially explicit observation

336 of community change through time rather than typical purely qualitative descriptions (Ferrari et

337 al. 2016a). Our ability to visualise and store the reef models will therefore allow verifiable and

338 archival observations, an increasingly sought after ability within the concept of reproducible

339 science (Munafò et al. 2017). 
340

341

342

343

344

345

346

347

348

350

351

352

353

354

355

356

357

358

359

360

\section{References}

Agisoft LLC. (2017). AgiSoft PhotoScan Professional edition (Version 1.3.4). Retrieved from http://www.agisoft.com/downloads/installer/.

Alvarez-Filip, L., Dulvy, N.K., Gill, J.A., Cote, I.M., Watkinson, A.R., Côté, I.M., Watkinson, A.R., Cote, I.M., Watkinson, A.R., Côté, I.M. \& Watkinson, A.R. (2009). Flattening of Caribbean coral reefs: region-wide declines in architectural complexity. Proceedings of the Royal Society of London B: Biological Sciences, 276, 3019-3025.

Bayley, D.T.I. \& Mogg, A.O.M. (2019). New Advances in Benthic Monitoring Technology and Methodology. World Seas: an Environmental Evaluation (ed C.R.C. Sheppard), pp. 121132. Elsevier.

Brock, J.C. \& Purkis, S.J. (2009). The Emerging Role of Lidar Remote Sensing in Coastal Research and Resource Management. Journal of Coastal Research, 10053, 1-5.

Brown, C.J., Smith, S.J., Lawton, P. \& Anderson, J.T. (2011). Benthic habitat mapping: A review of progress towards improved understanding of the spatial ecology of the seafloor using acoustic techniques. Estuarine, Coastal and Shelf Science, 92, 502-520.

Bryson, M., Ferrari, R., Figueira, W., Pizarro, O., Madin, J., Williams, S. \& Byrne, M. (2017). Characterization of measurement errors using structure-from-motion and photogrammetry to measure marine habitat structural complexity. Ecology and Evolution, 1-13.

Burns, J.J.H.R.R., Delparte, D., Gates, R.D. \& Takabayashi, M. (2015a). Integrating structurefrom-motion photogrammetry with geospatial software as a novel technique for quantifying 3D ecological characteristics of coral reefs. PeerJ, 3, e1077. 
361 Burns, J., Delparte, D., Gates, R. \& Takabayashi, M. (2015b). Integrating structure-from-motion

362 photogrammetry with geospatial software as a novel technique for quantifying 3D

363 ecological characteristics of coral reefs. PeerJ, 3, e1077.

364 Chirayath, V. \& Earle, S.A. (2016). Drones that see through waves - preliminary results from

365 airborne fluid lensing for centimetre-scale aquatic conservation. Aquatic Conservation:

$366 \quad$ Marine and Freshwater Ecosystems, 26, 237-250.

367 Costa, B.M., Battista, T.A. \& Pittman, S.J. (2009). Comparative evaluation of airborne LiDAR

368 and ship-based multibeam SoNAR bathymetry and intensity for mapping coral reef

369 ecosystems. Remote Sensing of Environment, 113, 1082-1100.

370

371

372

373

374

375

376

377

378

379

380

381

Darling, E.S., Graham, N.A.J., Januchowski-Hartley, F.A., Nash, K.L., Pratchett, M.S. \& Wilson, S.K. (2017). Relationships between structural complexity, coral traits, and reef fish assemblages. Coral Reefs.

Dustan, P., Doherty, O. \& Pardede, S. (2013). Digital Reef Rugosity Estimates Coral Reef Habitat Complexity. PLoS ONE, 8, 1-10.

English, S.A., Baker, V.J., Wilkinson, C.R. \& Wilkinson, C.R. (1997). Survey Manual for Tropical Marine Resources. Australian Institute of Marine Science.

Ferrari, R. (2017). The hidden structure in coral reefs. Coral Reefs, 36, 445.

Ferrari, R., Bryson, M., Bridge, T., Hustache, J., Williams, S.B., Byrne, M. \& Figueira, W. (2016a). Quantifying the response of structural complexity and community composition to environmental change in marine communities. Global Change Biology, 22, 1965-1975.

Ferrari, R., McKinnon, D., He, H., Smith, R.N., Corke, P., González-Rivero, M., Mumby, P.J. \& 
384 Figueira, W., Ferrari, R., Weatherby, E., Porter, A., Hawes, S. \& Byrne, M. (2015). Accuracy

385 and Precision of Habitat Structural Complexity Metrics Derived from Underwater 386 Photogrammetry. Remote Sensing, 7, 16883-16900.

387 Goatley, C.H.R. \& Bellwood, D.R. (2011). The Roles of Dimensionality, Canopies and 388 Complexity in Ecosystem Monitoring (H. Browman, Ed.). PLoS ONE, 6, e27307.

389 Graham, N.A.J., Jennings, S., Macneil, M.A., Mouillot, D. \& Wilson, S.K. (2015). Predicting 390 climate-driven regime shifts versus rebound potential in coral reefs. Nature, 518, 1-17.

391 Graham, N. a J. \& Nash, K.L. (2013). The importance of structural complexity in coral reef 392 ecosystems. Coral Reefs, 32, 315-326.

393 Harborne, A.R., Mumby, P.J. \& Ferrari, R. (2012). The effectiveness of different meso-scale 394 rugosity metrics for predicting intra-habitat variation in coral-reef fish assemblages. Environmental Biology of Fishes, 94, 431-442. Carreras, M. (2016). Autonomous underwater navigation and optical mapping in unknown natural environments. Sensors (Switzerland), 16.

Hitchin, R., Turner, J.A. \& Verling, E. (2015). Epibiota Remote Monitoring from Digital Imagery: Operational Guidelines. Joint Nature Conservation Committee. of seabed-mapping technologies in the context of marine habitat classification. ICES 

Journal of Marine Science, 60, 411-418.

404 Knudby, A. \& LeDrew, E. (2007). Measuring Structural Complexity on Coral Reefs.

405 Proceedings of the American Acadamy of Underwater Sciences 26th Symposium, 181-188.

406 Lavy, A., Eyal, G., Neal, B., Keren, R., Loya, Y. \& Ilan, M. (2015). A quick, easy and non-

407 intrusive method for underwater volume and surface area evaluation of benthic organisms

408 by 3D computer modelling (S. McMahon, Ed.). Methods in Ecology and Evolution, 6, 521409531.

410

411

412

413

414

415

416

417

418

419

420

421

422

423

Leon, J.X., Roelfsema, C.M., Saunders, M.I. \& Phinn, S.R. (2015). Measuring coral reef terrain roughness using 'Structure-from-Motion' close-range photogrammetry. Geomorphology, 242, 21-28.

Munafò, M.R., Nosek, B.A., Bishop, D.V.M., Button, K.S., Chambers, C.D., Percie, N., Simonsohn, U., Wagenmakers, E., Percie du Sert, N., Simonsohn, U., Wagenmakers, E., Ware, J.J. \& Ioannidis, J.P.A. (2017). A manifesto for reproducible science. Nature Human Behaviour, 1, 21.

Nash, K.L., Graham, N.A.J., Wilson, S.K. \& Bellwood, D.R. (2013). Cross-scale Habitat Structure Drives Fish Body Size Distributions on Coral Reefs. Ecosystems, 16, 478-490.

Nečas, D. \& Klapetek, P. (2012). Gwyddion: an open-source software for SPM data analysis. Open Physics, 10.

Perry, C.T., Edinger, E.N., Kench, P.S., Murphy, G.N., Smithers, S.G., Steneck, R.S. \& Mumby, P.J. (2012). Estimating rates of biologically driven coral reef framework production and erosion: a new census-based carbonate budget methodology and applications to the reefs of 
425 Pizarro, O., Friedman, A., Bryson, M., Williams, S.B. \& Madin, J. (2017). A simple, fast, and 426 repeatable survey method for underwater visual 3D benthic mapping and monitoring. 427 Ecology and Evolution, 7, 1770-1782.

428 Polunin, N. \& Roberts, C. (1993). Greater biomass and value of target coral-reef fishes in two 429 small Caribbean marine reserves. Marine Ecology Progress Series, 100, 167-176.

430 Purkis, S.J. (2018). Remote Sensing Tropical Coral Reefs: The View from Above. Annual 431 Review of Marine Science, 10, annurev-marine-121916-063249.

432 R Core Team. (2016). R: A Language and Environment for Statistical Computing.

433 Raoult, V., Reid-Anderson, S., Ferri, A. \& Williamson, J. (2017). How Reliable Is Structure 434 from Motion (SfM) over Time and between Observers? A Case Study Using Coral Reef 435 Bommies. Remote Sensing, 9, 740.

Risk, M.J. (1972). Fish Diversity on a Coral Reef in the Virgin Islands. Atoll Research Bulletin, $437 \quad 153,1-4$.

Robert, K., Huvenne, V.A.I., Georgiopoulou, A., Jones, D.O.B., Marsh, L., Carter, G. \& Chaumillon, L. (2017). New approaches to high-resolution mapping of marine vertical structures. Scientific Reports, 7, 9005.

441 Storlazzi, C.D., Dartnell, P., Hatcher, G.A. \& Gibbs, A.E. (2016). End of the chain? Rugosity 442 and fine-scale bathymetry from existing underwater digital imagery using structure-from443 motion (SfM) technology. Coral Reefs, 35, 889-894.

444 Teague, J. \& Scott, T. (2017). Underwater Photogrammetry and 3D Reconstruction of 
445 Submerged Objects in Shallow Environments by ROV and Underwater GPS. Journal of $446 \quad$ Marine Science Research and Technology.

447 De Waele, J., Fabbri, S., Santagata, T., Chiarini, V., Columbu, A. \& Pisani, L. (2018).

448 Geomorphological and speleogenetical observations using terrestrial laser scanning and 3D 449 photogrammetry in a gypsum cave (Emilia Romagna, N. Italy). Geomorphology, 319, 47$450 \quad 61$.

451 Westoby, M.J., Brasington, J., Glasser, N.F., Hambrey, M.J. \& Reynolds, J.M. (2012).

452 'Structure-from-Motion' photogrammetry: A low-cost, effective tool for geoscience 453 applications. Geomorphology, 179, 300-314.

454 Wilson, S.K., Graham, N.A.J. \& Polunin, N.V.C. (2007). Appraisal of visual assessments of 455 habitat complexity and benthic composition on coral reefs. Marine Biology, 151, 1069$456 \quad 1076$.

457 Young, G.C., Dey, S., Rogers, A.D. \& Exton, D. (2017). Cost and time-effective method for 458 multiscale measures of rugosity, fractal dimension, and vector dispersion from coral reef 3D 459 models. PLoS ONE, 12, 1-18. 
Figure 1

Location of the six reef sites surveyed along the Danajon Bank reef complex, north of Bohol, Philippines.

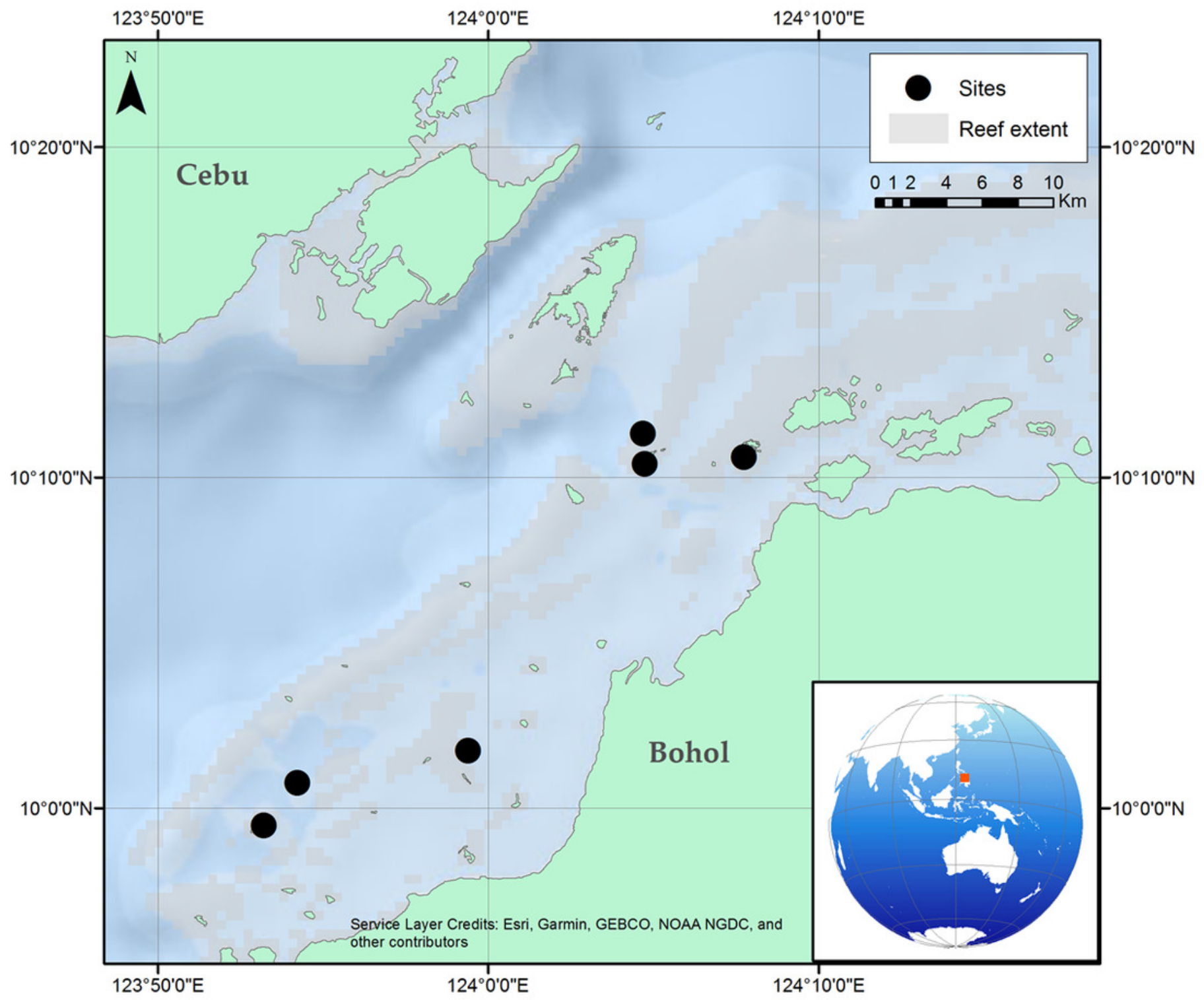


Figure 2

A virtual reef with Rugosity Index transects.

(A) Visualisation of linear and surface distance measurements across a typical $10 \times 1$ metre cross-section of reef. (B) A DEM of a medium rugosity mixed growth form Philippine reef, illustrating the layout of a $\sim 100 \mathrm{~m}^{2}$ benthic quadrat with transects (scale and depth shown). (C) The ten digital rugosity transects shown in section B, each with a $10 \mathrm{~m}$ Surface Distance. 

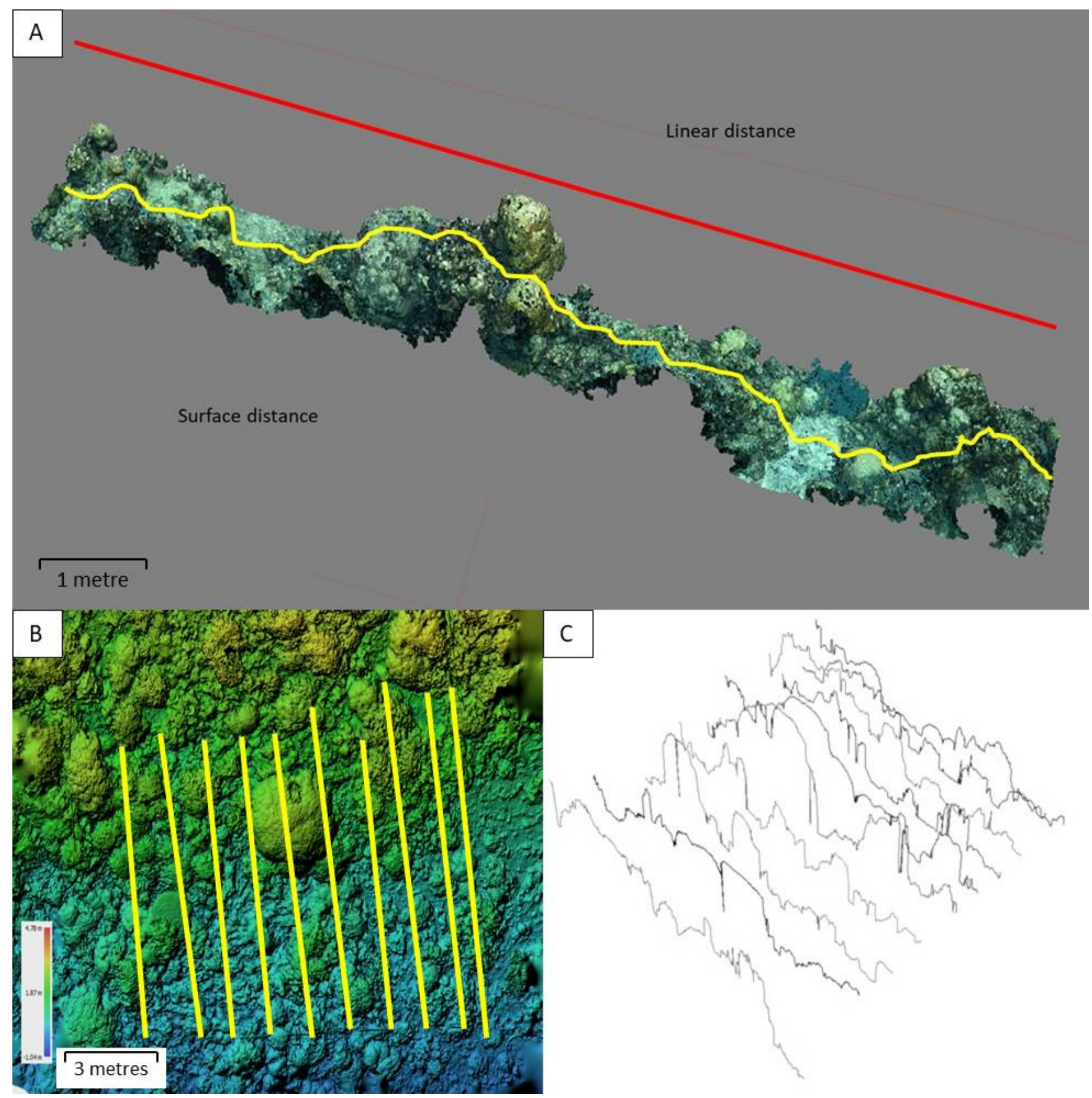


\section{Figure 3}

A $25 \mathrm{~m}^{2}$ reef area displayed within 'Gwyddion' software.

(A) XYZ pointcloud shown in false colour, with a white $5 \mathrm{~m}$ 'virtual transect' length selected.

(B) 'Roughness', 'waviness' (at $0.2 \mathrm{~m}$ sampling frequency cut-off) and 'texture' of the virtual transect surface. (C) Overall texture (rugosity) of the transect - equivalent to 'Surface Distance' at $1 \mathrm{~cm}$ resolution.

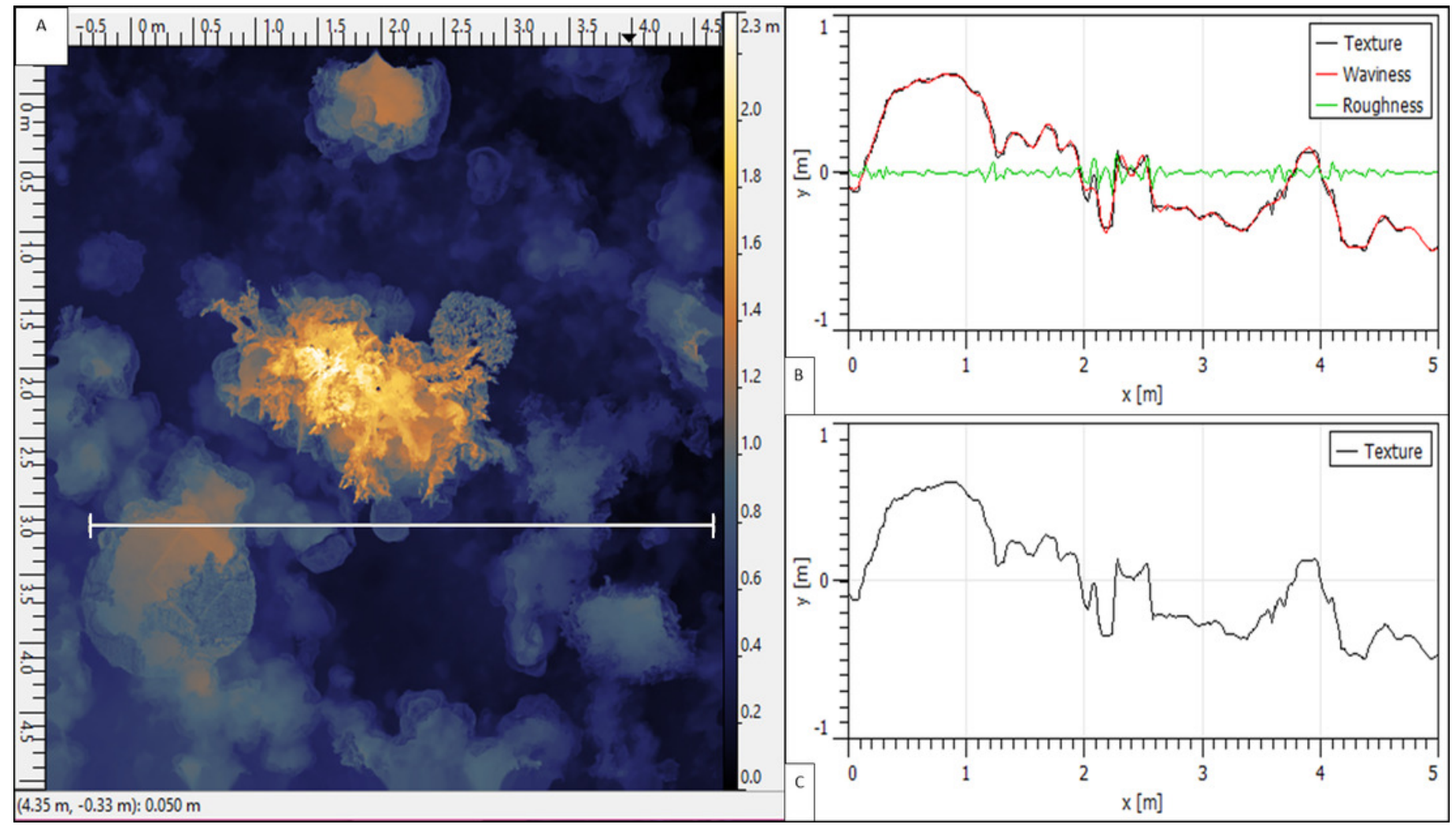




\section{Figure 4}

The difference between in-situ chain measured surface distance $(10 \mathrm{~m})$ and virtually measured surface distance, within five broad substrate types.

$A=$ Flattened rubble,$B=$ Foliose growth forms,$C=$ Table and massive growth forms on

sand, $D=$ Sparse corymbose and massive growth forms, $E=$ Dense branching coral growth forms. 


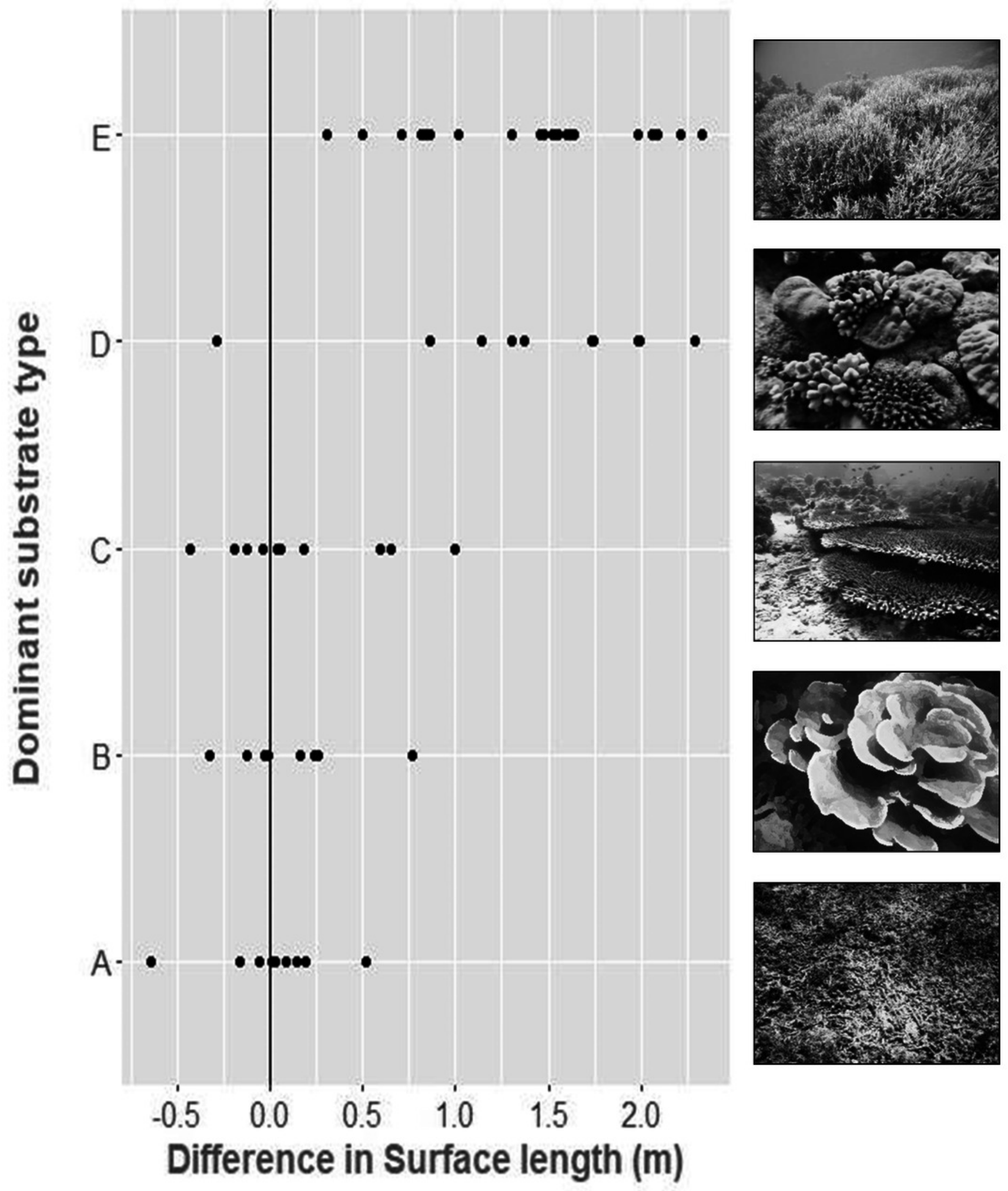


Figure 5

In situ chain-measured Rugosity Index against virtual SfM-derived Rugosity Index

The blue line shows the linear relationship between the measures (95\% Confidence Intervals shown). A dashed reference line (slope $=1$, intercept $=0$ ) is also shown for reference.

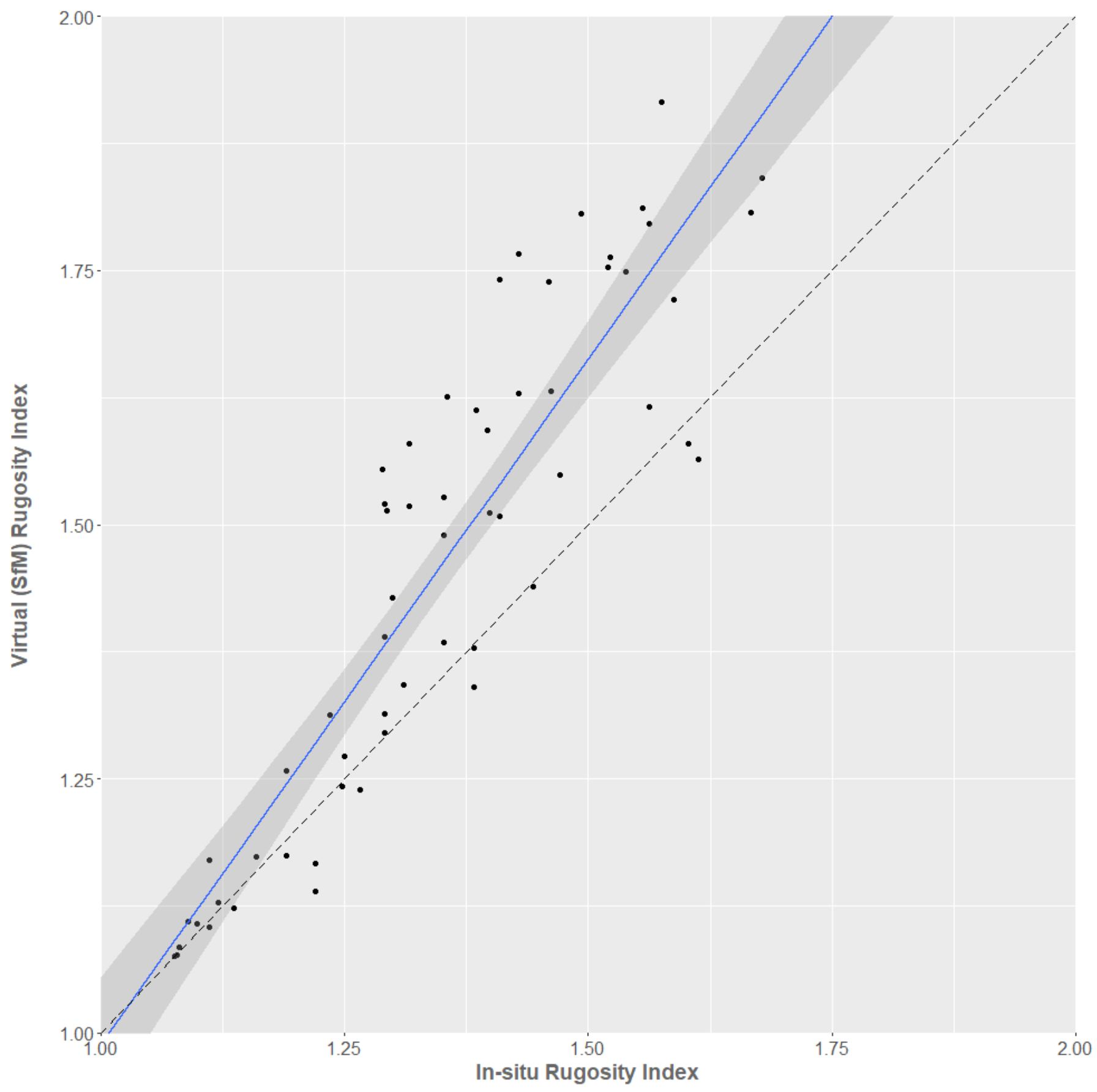


Figure 6

Comparison between (Polunin \& Roberts, 1993) visual method of structural assessment and the 'tape and chain' / SfM Rugosity Index methods

(A) Virtual SfM rugosity method against the visual assessment method. (B) In-situ tape and chain rugosity method against the visual assessment method. Individual data points overlaid and $95 \%$ Confidence Intervals shown in grey.

A

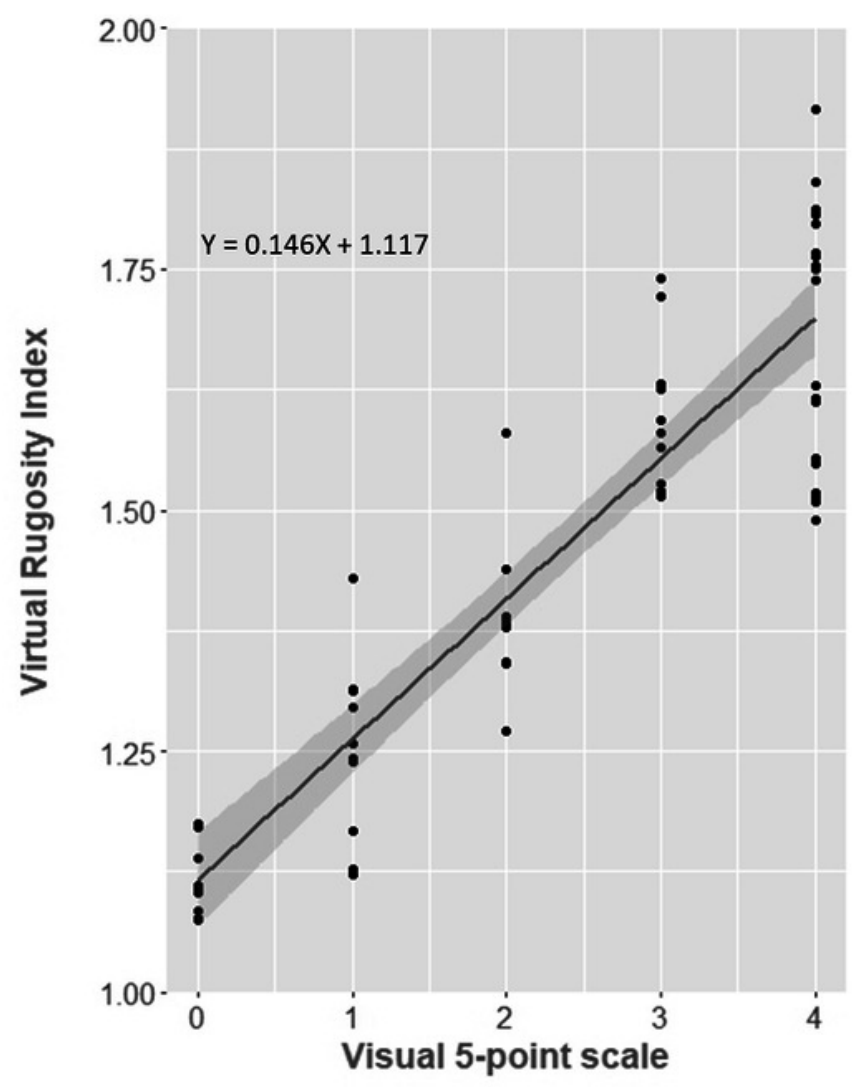

B

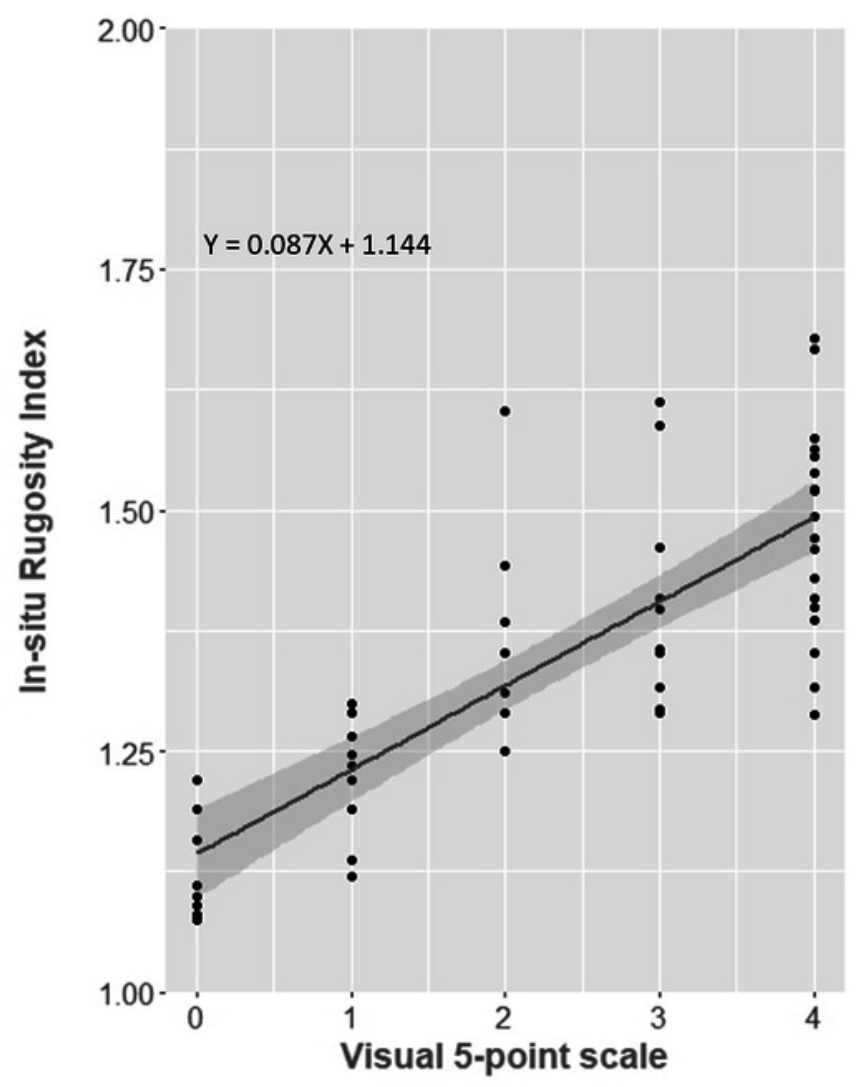




\section{Table $\mathbf{1}$ (on next page)}

Cost-benefits of four rugosity assessment techniques for analysing an area of $100 \mathrm{~m}^{2}$ or for ten $(10 \mathrm{~m})$ transects. 
1 Cost-benefits of four coral reef structure assessment techniques for analysing an area of $\sim 100 \mathrm{~m} 2$

2 (using Structure from Motion (SfM) or visual techniques), or for ten (10 m) in situ transects

3 using the 'tape and chain' Rugosity Index technique. SfM processing time based on $\sim 400$

4 images within Agisoft Photoscan run with a 32GB RAM, Intel Core i7 processor, and NVIDIA

5 GeForce GTX 960 graphical processor. The cluster used three nodes, each with the above stated

6 specifications.

\begin{tabular}{llllll}
\hline Technique & $\begin{array}{l}\text { Field } \\
\text { time }\end{array}$ & $\begin{array}{l}\text { Processing } \\
\text { time }\end{array}$ & Repeatable & $\begin{array}{l}\text { Other } \\
\text { analyses } \\
\text { possible? }\end{array}$ & $\begin{array}{l}\text { Remote } \\
\text { assessment } \\
\text { possible? }\end{array}$ \\
\hline $\begin{array}{l}\text { Tape and } \\
\text { chain }(\mathrm{x} \mathrm{10})\end{array}$ & $<1 \mathrm{hr}$ & None & No & No & No \\
Visual & $\begin{array}{l}< \\
\text { mins }\end{array}$ & None & No & No & Yes \\
SfM & $\begin{array}{l}30 \mathrm{mins} \\
-1 \mathrm{hr}\end{array}$ & $\sim 1$ day & Yes & Yes & Yes \\
$\begin{array}{l}\text { SfM-cluster } \\
\text { computing }\end{array}$ & $\begin{array}{l}30 \mathrm{mins} \\
-1 \mathrm{hr}\end{array}$ & $<1 \mathrm{hr}$ & Yes & Yes & Yes \\
\hline
\end{tabular}

7 